\title{
Electrical Signals of Memory and of the Awareness of Remembering
}

\author{
Ken A. Paller \\ Northwestern University
}

\begin{abstract}
Learning factual information and accurately remembering specific experiences from the past are central to human intellectual and social life. These extraordinary abilities require computations on diverse sorts of information represented in the brain. Networks of neurons in the cerebral cortex are specialized for analyzing and representing such information, whereas the storage of facts and events within these networks depends fundamentally on linking multiple representational fragments together. This cross-cortical linking function is disrupted in patients with amnesia. Electrical measures of the brain in action, obtained while people perform feats of memory in laboratory settings, have been used to investigate the storage and retrieval of facts and episodes. Electrical signals associated with specific aspects of memory processing have been identified through research that constitutes part of a larger scientific endeavor aimed at understanding memory, the subjective experience of remembering that can accompany retrieval, and disorders of memory that can result from brain damage.
\end{abstract}

KEYWORDS-declarative memory; priming; event-related potentials; ERPs; amnesia

What happens in your brain to allow you to remember a recent acquaintance, your favorite film, your last summer vacation, or your first kiss? Contemporary investigations of such phenomena are founded on systems for classifying types of memory. Many investigators focus on the category known as declarative memory, the ability to remember prior autobiographical episodes and complex facts (Squire, 1987). This ability can be demonstrated when an individual either retrieves learned information in a recall test or discriminates learned information from new information in a recognition test. Declarative memory provides each of us with a vast but imperfect storehouse of information, and a basis for our own life story.

Address correspondence to Ken Paller, Department of Psychology, Northwestern University, 2029 Sheridan Rd., Evanston, IL 602082710; e-mail: kap@northwestern.edu.
What would constitute a comprehensive scientific understanding of declarative memory? Relevant research concerning declarative memory spans the gamut from neurobiological studies in animals to cognitive modeling in computers. A long-standing and venerable approach to exploring both the neural and the psychological underpinnings of memory is to investigate memory deficits in neurological patients.

\section{NEUROCOGNITIVE FOUNDATIONS OF DECLARATIVE MEMORY}

Patients with a selective memory dysfunction and otherwise preserved intellectual functions are uncommon, but analyses of such cases have been extraordinarily informative. These amnesic patients generally have impairments in declarative memory, but not in certain other categories of memory, as listed in Table 1. Selective deficits in these patients imply that certain neural computations are essential for recalling and recognizing episodes and facts, but not for perceiving and manipulating the same sorts of information in other ways. An amnesic patient may carry on an intelligent and detailed conversation but, shortly afterward, be unable to remember that the conversation ever occurred.

Networks of neurons in the cerebral cortex (see Fig. 1) play a major role in perceiving and manipulating the information inherent in an episode, and alterations in connections between neurons in these same networks are thought to be responsible for declarative memory storage. A contemporary explanation for the disruption of declarative memory in amnesia postulates a core defect in a process of crosscortical storage - the process whereby the fragments of an episode or the various features of a complex fact become connected together into a coherent and sturdy representation in the brain (Paller, 2002). For example, fragments linked together in the cerebral cortex to form an enduring memory for an episode might include representations of sights, sounds, smells, a spatial layout of objects, people, actions, emotional coloring, a set of precipitating events, consequences of the episode, and so on. Representations of these different features are thought to depend on different cortical regions.

Storing declarative memories thus depends on linking cognitive representations instantiated not in a single brain region, but rather in many cortical networks specialized for different computations. The 
TABLE 1

Neuropsychological Findings in Patients With Selective Deficits in Declarative Memory

\begin{tabular}{|c|c|c|}
\hline Type of memory & Definition & Findings in amnesia \\
\hline Declarative memory & $\begin{array}{l}\text { Recall and recognition of episodes and facts } \\
\text { (i.e., episodic memory and semantic memory) }\end{array}$ & $\begin{array}{l}\text { Impairment in storage, producing deficits in } \\
\text { new learning (anterograde amnesia) and in } \\
\text { remembering information acquired prior to the } \\
\text { illness or injury (retrograde amnesia) }\end{array}$ \\
\hline Immediate memory & $\begin{array}{l}\text { Information kept in mind by continuous rehearsal } \\
\text { (e.g., verbal working memory) }\end{array}$ & Preserved \\
\hline Nondeclarative memory & & $\begin{array}{l}\text { Generally preserved, but with some notable } \\
\text { exceptions }\end{array}$ \\
\hline Perceptual priming & $\begin{array}{l}\text { Speeded or more accurate response to a specific stimulus, } \\
\text { as a result of altered perceptual representations }\end{array}$ & $\begin{array}{l}\text { Preserved if performance is not contaminated } \\
\text { by declarative memory (i.e., not based on episodic } \\
\text { retrieval during the implicit memory test) }\end{array}$ \\
\hline Conceptual priming & $\begin{array}{l}\text { Speeded or more accurate response to a specific stimulus, } \\
\text { as a result of altered conceptual representations }\end{array}$ & $\begin{array}{l}\text { Preserved in some cases, but further investigation is } \\
\text { required, particularly across stimulus domains }\end{array}$ \\
\hline Skills & $\begin{array}{l}\text { Behaviors that improve gradually with practice, } \\
\text { including cognitive skills (e.g., reading } \\
\text { mirror-reversed text) and motor skills }\end{array}$ & $\begin{array}{l}\text { Preserved when skill acquisition is } \\
\text { accomplished without reliance on declarative } \\
\text { memory (which is generally not the case for } \\
\text { typical skills learned in everyday settings) }\end{array}$ \\
\hline Classical conditioning & $\begin{array}{l}\text { Learned associations between two stimuli, one of which } \\
\text { elicits an automatic response }\end{array}$ & $\begin{array}{l}\text { Generally preserved, especially when conditioned } \\
\text { and unconditioned stimuli overlap temporally }\end{array}$ \\
\hline
\end{tabular}

Note. Not all subtypes of nondeclarative memory are listed.

most fundamental characteristic of declarative memories is postulated to be their dependence on representations in multiple cortical zones that must be linked together. Although much remains to be learned about this process, memory binding is thought to be accomplished through mechanisms that alter the interconnectivity of cortical neurons through interactions with other brain regions (such as the hippocampus, adjacent cortex of the medial temporal region, and portions of the thalamus). Indeed, amnesia often results from damage to the hippocampus, medial temporal region, or thalamus.

Furthermore, cross-cortical storage is not finalized immediately following a learning episode, but rather, it can evolve over an extended time course as the information becomes integrated with knowledge already accrued, as well as with information acquired subsequently. This process of cross-cortical consolidation may continue for many years for a fact or event that is reevaluated, reinterpreted, and repeatedly integrated with other information. Cross-cortical consolidation of a declarative memory may proceed not only during waking, but during sleep as well. It may even continue beyond a point when the memory has become cortically self-sufficient, which is when critical storage sites in the cortex can support retrieval of the memory even if the hippocampus and adjacent structures are dysfunctional. Such brain damage leads to difficulties remembering declarative memories that are not cortically self-sufficient, including memories formed prior to the onset of amnesia (retrograde amnesia) and memories formed after the onset of amnesia (anterograde amnesia). Because memories are less likely to be cortically self-sufficient the more recently they were acquired, retrograde amnesia is typically worse for recently acquired information than for older information. Many amnesic patients can remember facts and episodes from their childhood and early adulthood as well as anyone else their age.

Normal declarative memory is a product of three stages of information processing. Encoding refers to the initial stage, when in- formation arrives in the brain following sensory analysis or via imagination. The term encoding has been used to refer to the input and comprehension of this information (which is not problematic for amnesic patients), as well as to the transformation of the experience into a memory (which is impaired in amnesia).

Declarative memory formation may not be finalized at initial encoding, but rather can continue over a prolonged storage period, when memory is subject to change, consolidation, interference, distortion, and forgetting. Storage denotes this second stage of information processing.

The final stage, retrieval, takes place when a declarative memory is accessed and used. Amnesic patients are generally able to retrieve some declarative memories, particularly those already consolidated to the point of cortical self-sufficiency. However, memory retrieval can be quite demanding and require effortful search strategies, such as when one successfully searches for a relatively insignificant childhood memory. In such cases, contributions from a division of the cerebral cortex called prefrontal cortex (see Fig. 1) are especially important with respect to conducting a systematic search, evaluating products of retrieval, escaping from the present moment to bring a prior experience to mind, maintaining information in mind, inhibiting the intrusion of irrelevant information, constructing a remembered experience based on retrieved information, evaluating each bit of retrieved information to decide if it is plausible and appropriate with respect to current goals, and so on. Accordingly, prefrontal damage by itself can lead to memory retrieval difficulties, and when combined with medial temporal damage can lead to exacerbated memory deficits.

Although amnesic patients exhibit significant impairments in declarative memory, they can be entirely normal when it comes to other types of memory. Besides memory based on continuous rehearsal of information just encoded (i.e., immediate memory), preserved memory is also found for the category of nondeclarative memory - which is 


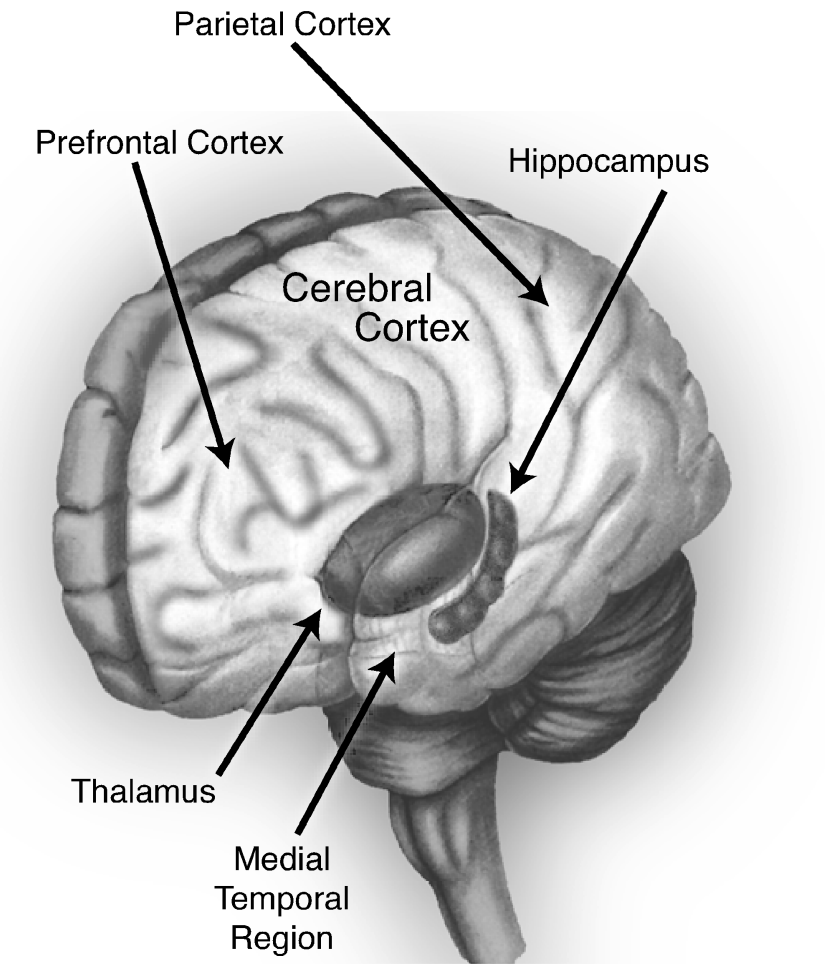

Fig. 1. Sketch of a human brain showing some of the brain regions involved in memory storage. The cerebral cortex is the large, outer portion of the brain with an infolded structure. It comprises two hemispheres, each of which includes parietal cortex at the top, prefrontal cortex behind the forehead, occipital cortex toward the back of the head, and temporal cortex at the side. The thalamus is a paired structure that would be hidden from view, but it can be seen on one side where the cortex is drawn as if it were transparent. Neurons in the thalamus are extensively interconnected with neurons in the cerebral cortex. The medial temporal region, which includes the hippocampus, would also be hidden from view but can be seen on one side through transparently illustrated temporal cortex. The hippocampus receives information indirectly from many cortical regions. Sensory information is analyzed in networks of neurons in occipital, parietal, and temporal cortex. Information relevant for remembering facts and episodes is represented in various cortical regions, and neurons in these regions are interconnected with each other and with neurons in the medial temporal region.

defined by exclusion as distinct from immediate memory and from declarative memory, and which is not accessible to conscious recollection (see Table 1 for examples). This evidence underscores the idea that declarative memory depends on special storage mechanisms. Nondeclarative memory differs from declarative memory in that it does not require the linking of distinct representations across multiple cortical zones. Often, tests of nondeclarative memory do not make explicit reference to prior learning episodes (such tests are called implicit memory tests, and memory demonstrated in these tests is sometimes called implicit memory). For instance, behavioral responses to a specific stimulus may be faster or more accurate as a result of prior experience, even when a person is unable to remember that prior experience. This behavioral effect constitutes priming, a key type of nondeclarative memory. Understanding the fundamental differences between declarative and nondeclarative memory can shed light on the neurocognitive mechanisms unique to declarative memory.
Furthermore, understanding special cases in which nondeclarative memory is not preserved in amnesia may provide pivotal insights into the core defect. Future tests of the conceptualization of declarative memory I have summarized here should determine whether priming is preserved in amnesia because of experience-induced neural changes within isolated cortical zones, and whether some subtypes of priming tend to be impaired in amnesia when priming requires changes in connections among neurons in different cortical zones.

\section{ELECTROPHYSIOLOGY OF DECLARATIVE MEMORY}

To gain further insight into the distinct cognitive functions that combine to support declarative memory, it will be crucial to be able to measure these functions independently. Indeed, electrical activity from the brain can be recorded noninvasively in healthy individuals, and relevant measures can be obtained on a millisecond-by-millisecond basis in order to test and advance theoretical proposals developed through neuropsychological studies of memory disorders.

The electroencephalogram (EEG) is a summation of electrical fields produced by activity in vast numbers of neurons and recorded using electrodes placed harmlessly on an individual's head. An eventrelated potential (ERP) is an average response to a class of events, such as one type of stimulus, and can be calculated by averaging EEG responses to multiple stimuli presented to the individual in a suitable experimental setting (see Friedman \& Johnson, 2000). ERPs can be characterized in terms of their latency (when they occur relative to the onset of a stimulus), their polarity (positive or negative at the recording location relative to a distant reference location), their amplitude (size of a potential deflection), and their topography (distribution of potential amplitudes across the head). In the following sections, I describe research from my laboratory associating certain memory functions with particular ERP signals. Despite this emphasis on ERP research, the general approach advocated here also applies to research with other direct and indirect measures of brain activity, including measures of blood flow, metabolism, and magnetic fields.

\section{TRANSFORMING EXPERIENCE INTO MEMORY}

One way to investigate the formation of declarative memories is to examine neural activity at initial encoding and determine which neural activity predicts successful versus unsuccessful memory performance. Brain potentials that predict successful subsequent recall and recognition have been observed in many experiments. These potentials are generally positive over parietal or prefrontal brain regions and reach maximal amplitudes 400 to $800 \mathrm{~ms}$ or so after stimulus onset, with larger amplitudes for remembered than forgotten stimuli. Similar ERPs were observed in a few experiments in which electrodes were implanted in the medial temporal region in patients who were candidates for surgery to relieve medically intractable epilepsy. ERPs that predict whether a person will remember seeing a common object have also been identified, as have ERPs that predict whether a person will claim to have seen an object that was not actually seen but rather was imagined. To-be-remembered stimuli in all these ERP studies have included objects, faces, spoken names, environmental sounds, and, most often, words.

In one experiment, words were presented visually in an encoding phase followed by either an implicit or an explicit memory test (Paller, 1990). In the implicit memory test, participants were instructed to 
complete three-letter stems with the first word to come to mind. The number of completions that matched words from encoding, compared with a baseline rate of such completions, provided a measure of priming. In the explicit memory test, participants attempted to recall words from the encoding phase in order to complete the stems. ERPs from the encoding phase were more positive for words later recalled on the explicit memory test than for words not recalled. This systematic difference in brain potentials can be referred to as Dm-recall (an ERP $D$ ifference based on later memory performance on the recall test). In contrast, ERPs did not reliably predict later priming. These findings, along with others, are consistent with the idea that Dm-recall indexed encoding activity specific to declarative memory formation, most likely processing pertaining to the meaning of each word rather than its visual appearance.

In an experiment with faces, ERPs at initial encoding predicted not only whether later recognition would be successful, but also the experiential quality of the recognition experience (Yovel \& Paller, 2004). Positive ERPs from parietal regions over both left and right hemispheres predicted successful recognition accompanied by retrieval of episodic detail, whereas only right-parietal ERPs predicted successful recognition without episodic detail, a phenomenon referred to as pure familiarity - when a face seems familiar but is not remembered.

Other studies of ERPs, electrical rhythms, functional magnetic resonance images of brain activity, and spiking from single neurons have suggested that many cortical regions can be involved in memory encoding and that activity in the hippocampus may be particularly relevant for the storage of declarative memories (e.g., Fell, Klaver, Elger, \& Fernandez, 2002; Paller \& McCarthy, 2002; Reber et al., 2002; Sederberg, Kahana, Howard, Donner, \& Madsen, 2003).

Many different types of processing at encoding can promote successful memory storage. Accordingly, many avenues of investigation will be required for scientists to understand the formation and preservation of declarative memories. Measures of neural activity predictive of subsequent memory, such as Dm-recall, provide an inroad to this problem, and will ultimately be most useful if connections can be built between these measures and specific neurocognitive processes. This goal will require analyzing neural activity as a function of successful versus unsuccessful encoding in conjunction with manipulating various factors that systematically affect memory encoding and storage.

\section{MEMORY RETRIEVAL}

The efficacy of encoding and storage becomes evident only when stored information is subsequently accessed. In studies of retrieval, differences between ERP responses to new and old items in recognition tests (i.e., items not previously presented and those presented at encoding, respectively) have been researched in considerable detail. These old-new ERP effects generally take the form of positive shifts in ERPs to old items relative to ERPs to new items.

Early experimental results prompted a range of conclusions regarding the cognitive concomitants of these effects without leading to consensus. In retrospect, firm interpretations were difficult because discriminating old from new generally involves a variety of different cognitive processes and multiple brain potentials that overlap in time. As a result, functionally distinct brain signals within old-new ERP effects were difficult to isolate from one another.
For example, consider two memory phenomena that can co-occur when a person views a face: (a) retrieval of prior episodes involving the same face and (b) faster or more accurate processing of that face due to prior perceptual analysis of the same face (the behavioral phenomenon of perceptual priming). Special tactics are needed to isolate ERPs associated with these different sorts of memory. Indeed, it is notoriously difficult to prevent people from systematically recalling prior episodes when stimuli are repeated, and this incidental retrieval can contaminate neural analyses of priming.

One approach to this problem made use of a condition in which faces were encoded only to a minimal extent (Paller, Hutson, Miller, \& Boehm, 2003). Each of these faces was presented at a central location for $100 \mathrm{~ms}$ while participants were required to make a difficult visual discrimination at another location. When the face disappeared, a masking stimulus appeared centrally to further limit face encoding. On a subsequent test, participants' ability to recognize these faces was nearly the same as would be expected if they were merely guessing which faces had been presented previously. However, priming was still observed for these faces in an implicit memory test. Thus, ERPs elicited by these faces were associated with priming uncontaminated by conscious remembering. In contrast, other faces were well remembered by the participants because they were initially presented for a longer duration and without the additional discrimination requirement that interfered with encoding. The two conditions thus provide a direct comparison between ERPs associated with conscious memory for faces and ERPs associated with priming. Recognizing a repeated face was associated with positive ERPs at the rear of the head 400 to $800 \mathrm{~ms}$ after face onset (Fig. 2a), whereas priming was associated with negative ERPs at the front of the head 200 to $400 \mathrm{~ms}$ after face onset (Fig. 2b).

In another experiment, we used a different strategy to isolate signals associated with face recollection (Paller, Bozic, Ranganath, Grabowecky, \& Yamada, 1999). At encoding, participants attempted to memorize 20 faces accompanied by spoken vignettes (simulating actually meeting the individuals pictured) and were told to forget 20 other faces. Later recognition was superior for the former compared with the latter faces, but the magnitude of priming observed during implicit memory testing was the same for the two groups of faces. Comparing ERPs for the two kinds of faces therefore revealed an uncontaminated neural signal of face recollection (comparable to that shown in Fig. 2a). In a subsequent experiment, similar electrical signals were observed for remembering a face per se and for remembering a face along with corresponding biographical facts learned at encoding. Brain activity was observed over posterior regions in both situations, whereas additional activity that was slightly more anterior was observed only when biographical retrieval occurred. Subjects in these experiments were able to successfully recall person-specific information, which is an ability that depends on linking diverse sorts of information together-a prototypical example of declarative memory retrieval that would naturally give rise to conscious recollection.

\section{AWARENESS OF REMEMBERING}

Despite the strong connection between declarative memory and the experience of remembering, these phenomena need not always occur together. Declarative memory retrieval provides some of the necessary precursors for the awareness of remembering, but it is not sufficient to 
A
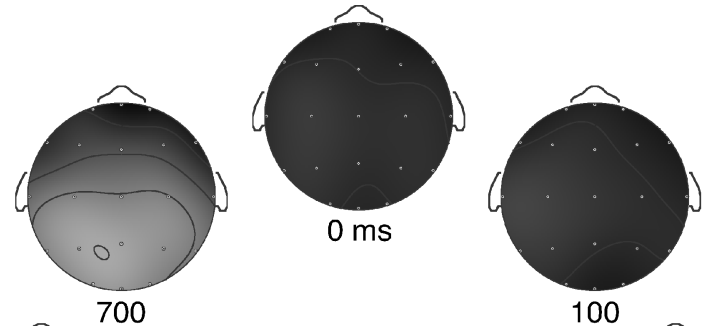

B
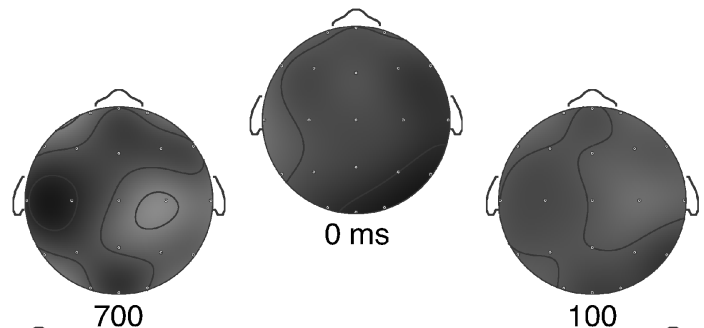

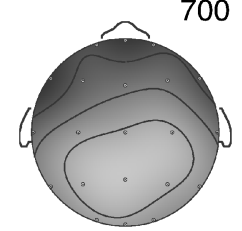

600

Recollection

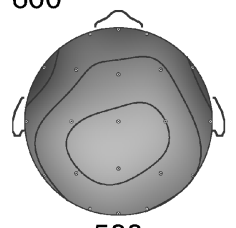

500
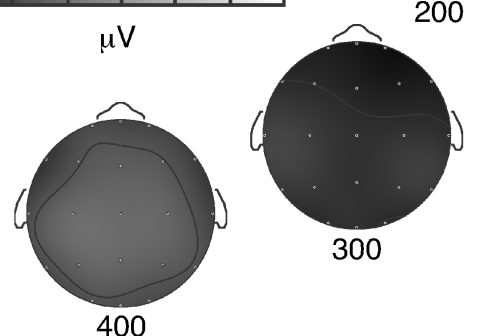

300

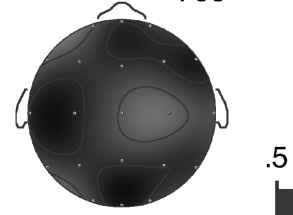

Priming

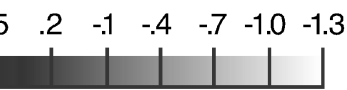

100

600

$\mu \mathrm{V}$

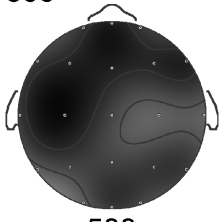

500
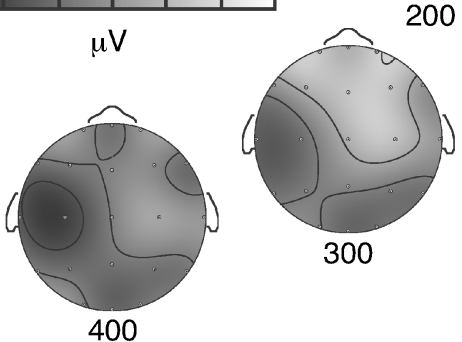

200

C

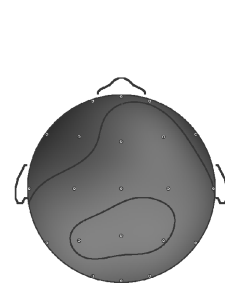

700
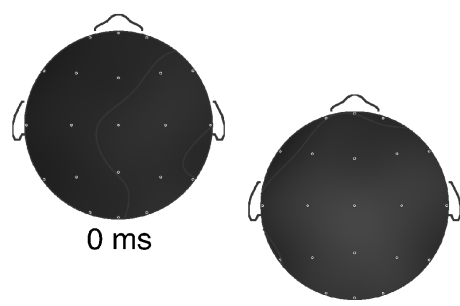

100

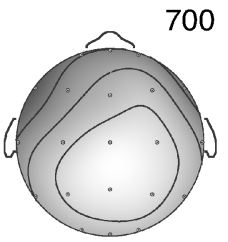

600

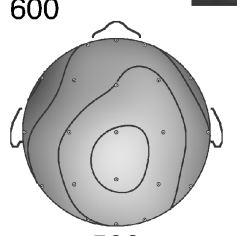

500
Recollection
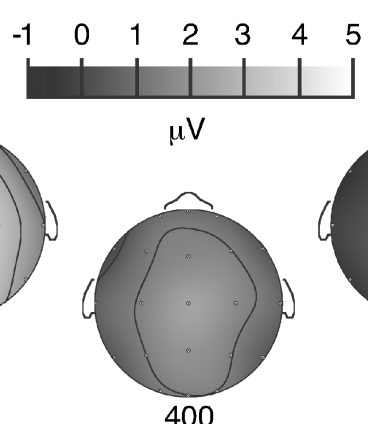

D
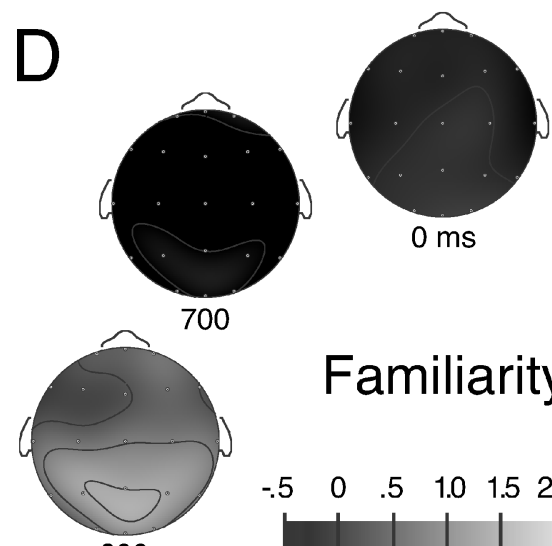

600

$\begin{array}{rrrrrrr}-.5 & 0 & .5 & 1.0 & 1.5 & 2.0 & 2.5\end{array}$

Familiarity

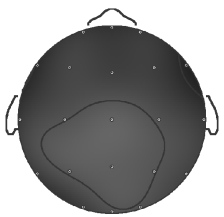

100
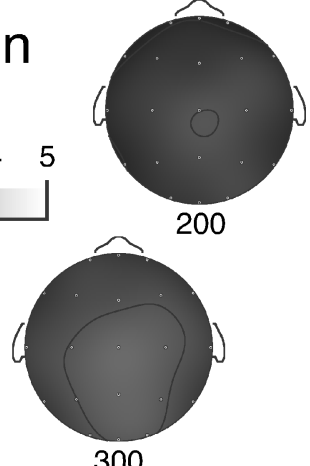

300
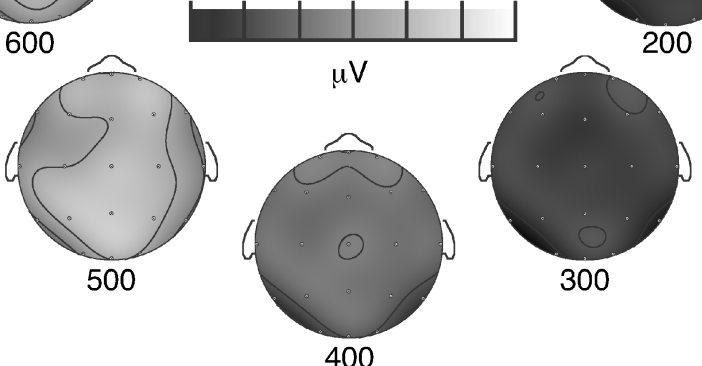

Fig. 2. Topographic maps of brain potentials associated with different memory experiences cued by faces: recollection (a, c), priming (b), and familiarity (d). In each panel, differences in potentials between two conditions are shown on schematic heads as if viewed from above (nose at the top), results are interpolated from 21 scalp locations (small circles), and measurements are displayed for eight intervals beginning at face onset ( 0 ms). Each of the eight maps represents a mean amplitude difference computed over a 100 -ms interval beginning at the time shown. The largest differences are signified by the lightest shades of gray, although the microvolt scale is not the same in each panel. In (b), differences are negative potentials, whereas in the other panels, differences are positive. In (d), values beyond the negative range of the scale in the map for 700 to $800 \mathrm{~ms}$ are shown in black. Results shown in (a) and (b) are adapted from Paller, Hutson, Miller, and Boehm (2003). The recollection contrast is between remembered faces and new faces; the priming contrast is between primed but not remembered faces and new faces. Results shown in (c) and (d) are from Yovel and Paller (2004). The recollection contrast is between faces recognized with episodic recollection and new faces; the familiarity contrast is between faces recognized without episodic recollection and new faces. Color topographic maps are available in the original publications. 
produce this experience. Rather, conscious memory depends on a further inference-the explicit thought that the current contents of consciousness are derived from memory retrieval.

Thus, dysfunctional cross-cortical storage in amnesia has an indirect impact on awareness of remembering. A strong, selective disruption of declarative memory also tends to disrupt awareness of remembering because memory for the spatiotemporal context of an episode is a critical factor that can help one to infer that a memory for a prior event has been retrieved (e.g., Johnson \& Chalfonte, 1994). The ability to make such inferences is one of several retrieval functions dependent on prefrontal cortex (along with strategic search, evaluation, and keeping retrieved information in mind). Indeed, retrieval functions have been associated with ERPs over prefrontal cortex in many studies (e.g., Ranganath \& Paller, 1999; Rugg \& Wilding, 2000).

Neural signals of memory must thus be evaluated with respect to the possibility that declarative memory retrieval need not necessarily give rise to awareness of remembering. That is, sometimes a stimulus can seem familiar even in the absence of conscious remembering. Data relevant to understanding the difference between retrieval with and without conscious remembering were recently obtained by comparing the case in which a face provoked a full-blown recollective experience with the case in which a face provoked the unsubstantiated impression of memory known as pure familiarity (Yovel \& Paller, 2004). Electrical signals associated with face-induced recollection (Fig. 2c) and face-induced pure familiarity (Fig. 2d) were similar, but amplitudes were reduced for pure familiarity. Notably, electrophysiological correlates of pure familiarity with faces and of priming with faces (Figs. 2d and Fig. 2b, respectively) were highly divergent, consistent with the notion that familiarity is not a straightforward outcome of priming, despite superficial similarities between familiarity and priming. Pure familiarity can instead be conceived of as a result of limited stimulus recognition without contextual retrieval adequate for triggering episodic recollection.

\section{BORDER AREAS OF DECLARATIVE MEMORY}

Current theories of memory address a variety of memory phenomena and their neural implementation, but many questions remain open. Some subtle but critical questions concern memory phenomena at the border between declarative and nondeclarative memory, such as several subtypes of priming. If amnesia fundamentally entails a disruption of memory functions dependent on cross-cortical storage, as proposed, then priming should remain preserved only if mediated within single cortical zones.

Conceptual priming is one subtype of priming that deserves further study; it is thought to arise from altered representations of the meaning of a stimulus rather than of the physical features of a stimulus. Conceptual priming can cross stimulus domains, such as when hearing a word primes its meaning so as to facilitate processing of the meaning when the word is subsequently read, or when reading the name of a famous person primes knowledge of his or her identity so as to facilitate processing of that person's identity when his or her face is subsequently viewed. Indeed, a putative electrical signal of conceptual priming with words was identified and shown to be preserved in patients with deficient declarative memory (Olichney et al., 2000; see also Yovel \& Paller, 2004). This signal may reflect a component of exactly the type of memory that allows amnesic patients to engage fully in complex conversations, all the while maintaining their comprehension abilities and focus on the topic at hand.

\section{CONCLUSIONS}

Researchers now have the ability to record neural signals associated with several of the many processes that contribute to declarative and nondeclarative memory. These neural signals provide a window into the physiology of memory that will be essential for further explorations of the neurocognitive substrates of remembering.

Future efforts should be aimed at elucidating exactly how declarative memory differs from priming and other sorts of nondeclarative memory. What is unique about declarative encoding, storage, and retrieval? What memory processes support priming, and how do these processes differ from those that support declarative memory? Does remembering in the absence of contextual retrieval, as exemplified by pure familiarity experiences, rely on any memory processing in common with priming? What processing underlies priming phenomena that are impaired in amnesia?

A promising strategy to promote progress on these and related issues is to isolate and characterize neurophysiological events specifically responsible for memory. A variety of techniques for measuring brain activity can be used together to study human memory and memory disorders, and to provide data needed to advance and refine neurobiological hypotheses concerning memory, such as those I have outlined in this review. This approach may also lead to an eventual understanding of how neurocognitive processing gives rise to the conscious experience of remembering, and it may thus also provide clues to understanding subjective awareness in general.

\section{Recommended Reading}

Eichenbaum, H., \& Cohen, N.J. (2001). From conditioning to conscious recollection: Memory systems of the brain. New York: Oxford University Press.

Münte, T.F., Urbach, T.P., Düzel, E., \& Kutas, M. (2000). Event-related brain potentials in the study of human cognition and neuropsychology. In F. Boller, J. Grafman, \& G. Rizzolatti (Eds.) Handbook of neuropsychology, Vol. 1 (pp. 139-234). Amsterdam: Elsevier-Science.

Paller, K.A. (1997). Consolidating dispersed neocortical memories: The missing link in amnesia. In A.R. Mayes \& J.J. Downes (Eds.), Theories of organic amnesia (pp. 73-88). East Sussex, England: Psychology Press. (Reprinted from Memory, 5, 73-88, 1997).

Paller, K.A., \& Wagner, A.D. (2002). Observing the transformation of experience into memory. Trends in Cognitive Sciences, 6, 93-102.

Schacter, D.L. (1996). Searching for memory: The brain, the mind, and the past. New York: Basic Books.

Squire, L.R., \& Kandel, E.R. (1999). Memory: From mind to molecules. New York: Scientific American Library.

Squire, L.R., \& Schacter, D.L. (Eds.). (2002). Neuropsychology of memory (3rd ed.). New York: Guilford.

Acknowledgments-Research reported in this article was supported by Grant NS34639 from the National Institute of Neurological Disorders and Stroke. I thank Gary Paller for adapting multiple images to construct Figure 1. 


\section{REFERENCES}

Fell, J., Klaver, P., Elger, C.E., \& Fernandez, G. (2002). The interaction of rhinal cortex and hippocampus in human declarative memory formation. Reviews in the Neurosciences, 13, 299-312.

Friedman, D., \& Johnson, R., Jr. (2000). Event-related potential (ERP) studies of memory encoding and retrieval: A selective review. Microscopy Research and Technique, 51, 6-28.

Johnson, M.K., \& Chalfonte, B.L. (1994). Binding complex memories: The role of reactivation and the hippocampus. In D.L. Schacter \& E. Tulving (Eds.), Memory systems 1994 (pp. 311-350). Cambridge MA: MIT Press.

Olichney, J.M., Van Petten, C., Paller, K.A., Salmon, D.P., Iragui, V.J., \& Kutas, M. (2000). Word repetition in amnesia: Electrophysiological measures of impaired and spared memory. Brain, 123, 1948-1963.

Paller, K.A. (1990). Recall and stem-completion priming have different electrophysiological correlates and are modified differentially by directed forgetting. Journal of Experimental Psychology: Learning, Memory, and Cognition, 16, 1021-1032.

Paller, K.A. (2002). Cross-cortical consolidation as the core defect in amnesia: Prospects for hypothesis-testing with neuropsychology and neuroimaging. In L.R. Squire \& D.L. Schacter (Eds.), Neuropsychology of memory (3rd ed., pp. 73-87). New York: Guilford.
Paller, K.A., Bozic, V.S., Ranganath, C., Grabowecky, M., \& Yamada, S. (1999). Brain waves following remembered faces index conscious recollection. Cognitive Brain Research, 7, 519-531.

Paller, K.A., Hutson, C.A., Miller, B.B., \& Boehm, S.G. (2003). Neural manifestations of memory with and without awareness. Neuron, 38, 507-516.

Paller, K.A., \& McCarthy, G. (2002). Field potentials in the human hippocampus during the encoding and recognition of visual stimuli. Hippocampus, 12, 415-420.

Ranganath, C., \& Paller, K.A. (1999). Frontal brain potentials during recognition are modulated by requirements to retrieve perceptual detail. Neuron, 22, 605-613.

Reber, P.J., Siwiec, R.M., Gitelman, D.R., Parrish, T.B., Mesulam, M.-M., \& Paller, K.A. (2002). Neural correlates of successful encoding identified using functional magnetic resonance imaging. Journal of Neuroscience, 22, 9541-9548.

Rugg, M.D., \& Wilding, E.L. (2000). Retrieval processing and episodic memory. Trends in Cognitive Sciences, 4, 108-115.

Sederberg, P.B., Kahana, M.J., Howard, M.W., Donner, E., \& Madsen, J.R. (2003). Theta and gamma oscillations during encoding predict subsequent recall. Journal of Neuroscience, 23, 10809-10814.

Squire, L.R. (1987). Memory and brain. New York: Oxford University Press.

Yovel, G., \& Paller, K.A. (2004). The neural basis of the butcher-on-the-bus phenomenon: When a face seems familiar but is not remembered. NeuroImage, 21, 789-800. 